\title{
Terahertz-wave Parametric Sources
}

\author{
Shin'ichiro Hayashi ${ }^{1}$ and Kodo Kawase ${ }^{1,2}$ \\ ${ }^{1}$ RIKEN, \\ ${ }^{2}$ Nagoya University \\ Japan
}

\section{Introduction}

Terahertz waves are the electromagnetic radiation whose frequency ranges from millimeter waves to the far infrared, shown in Figure 1. While both sides of this range have had a long history of research and development, leading to already commercially available sources, detectors, meters, and many additional devices, the terahertz wave range is still in its infancy, representing the last unexplored part of the electromagnetic spectrum between radio waves and visible light. This delayed development was mainly caused by the difficulty of producing reliable and strong terahertz wave generators, as well as the unavailability of sensors that can detect this unusual radiation. Technology extrapolation from both neighboring sides has been facing difficult problems: Up-conversion from the microwaves is inefficient due to the frequency being too high; down-conversion from the infrared is limited by the energy gaps.

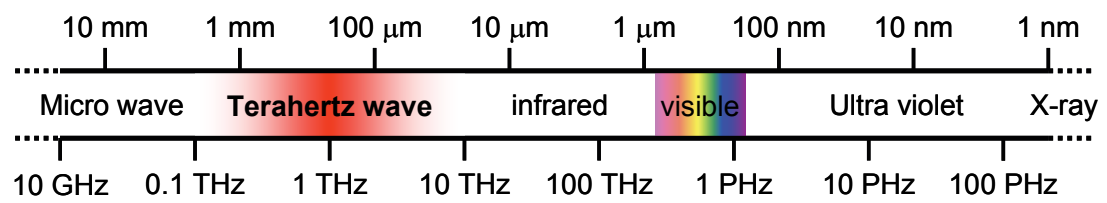

Fig. 1. A schematic showing the terahertz wave within the electromagnetic spectrum.

In recent years, terahertz wave sources have received considerable attention for use in many applications. Especially, recent research using terahertz waves, transmission imaging and fingerprint spectra have had an important contribution in the bioengineering and security fields, such as in material science, solid state physics, molecular analysis, atmospheric research, biology, chemistry, drug and food inspection, and gas tracing (Tonouchi, 2007).

There are several ways to generate terahertz waves. In the laboratory, one of the most widespread processes is the optical rectification or photoconductive switching produced using femtosecond laser pulses (Smith et al., 1988; Zhang et al., 1990). Applied research, such as time domain spectroscopy (THz-TDS), makes use of the good time resolution and the ultra broad bandwidth, up to the terahertz region. Novel tunable sources already exist in the sub-THz (several hundred GHz) frequency region, such as the backward-wave oscillator (BWO). However, the output power of a BWO rapidly decreases in the frequency region above $1 \mathrm{THz}$, and its tuning capability is relatively limited. 
Only few sources bring together qualities such as room temperature operation, compactness, and ease of use. The terahertz wave parametric generation is based on an optical parametric process in a nonlinear crystal (Sussman, 1970; Pietrup et al., 1975). The principles of the terahertz wave parametric generator (TPG) (Shikata et al., 2000; Sato et al., 2001; Shikata et al., 2002) and the terahertz wave parametric oscillator (TPO) (Kawase et al., 1996; 1997; 2001) allow building systems that are not only compact but also operate at room temperature, making them suitable as practical sources. In principle, both a narrow linewidth and a wide tunability are possible in injection-seeded TPG/TPO (is-TPG/TPO) systems with single-longitudinal-mode near-infrared lasers as seeders (Kawase et al., 2001; 2002; Imai et al., 2001).

In basic research, these sources were pumped using flash lamp- or laser diode- pumped Qswitched Nd:YAG lasers which have Gaussian beam profile and long pulse widths $(15 \sim 25$ ns). The output energy of terahertz wave increases with the pump energy, but eventually the damage threshold of the crystals is reached. Recently, we demonstrated how the output energy/power was further enhanced and how the TPG was reduced to palmtop size by using a small pump source having a short pulse width and top-hat beam profile (Hayashi et al., 2007). These characteristics of the pump beam enable high intensity pumping especially close to the output surface of the terahertz wave without thermal damage of the crystal surface. The higher intensity pumping and smaller absorption of the terahertz wave inside the crystal enable higher output energy than previously reported. Further, we also demonstrated a compact and tunable terahertz wave parametric source pumped by a microchip Nd:YAG laser, seeded with the idler wave provided by an external cavity diode laser (ECDL) (Hayashi et al., 2009). We show how the output peak power and tunability were further enhanced and how the is-TPG was reduced to palmtop size by using a passively Q-switched small pump source having a short pulse width. These characteristics of the pump beam permit high intensity pumping close to the output surface of the terahertz wave without thermal damage to the crystal surface. The higher intensity pumping and smaller absorption of the terahertz wave inside the crystal allow a broader tuning range than that previously reported.

\section{Principles of a Terahertz-wave parametric generation}

When a strong laser beam propagates through a nonlinear crystal, photon and phonon transverse wave fields are coupled, behave as new mixed photon-phonon states, called polaritons. The generation of the terahertz wave results from the efficient parametric scattering of laser light via a polariton, that is, stimulated polariton scattering. The scattering process involves both second- and third-order nonlinear processes. Thus, strong interaction occurs among the pump beam, the idler wave, and the polariton (terahertz) wave.

One of the most suitable nonlinear crystal to generate terahertz wave is the lithium niobate $\left(\mathrm{LiNbO}_{3}\right)$ thanks to its large nonlinear coefficient $\left(\mathrm{d}_{33}=25.2 \mathrm{pmV}^{-1}\right.$ at $\left.\lambda=1064 \mathrm{~nm}\right)$ (Shoji et al., 1997) and its transparency over a wide wavelength range $(0.4-5.5 \mu \mathrm{m})$. $\mathrm{LiNbO}_{3}$ has four infrared- and Raman-active transverse optical (TO) phonon modes, called $\mathrm{A}_{1}$-symmetry modes, and the lowest mode $\left(\omega_{0} \sim 250 \mathrm{~cm}^{-1}\right)$ is useful for efficient terahertz wave generation because it has the largest parametric gain as well as the smallest absorption coefficient.

The principle of tunable terahertz wave generation is as follows. The polaritons exhibit phonon-like behavior in the resonant frequency region (near the TO-phonon frequency $\omega_{\mathrm{TO}}$ ). However, they behave like photons in the non resonant low-frequency region as shown in 
Figure 2, where a signal photon at terahertz frequency $\left(\omega_{T}\right)$ and a near-infrared idler photon $\left(\omega_{i}\right)$ are created parametrically from a near-infrared pump photon $\left(\omega_{p}\right)$, according to the energy conservation law $\omega_{p}=\omega_{T}+\omega_{i}$ (p: pump beam, T: terahertz wave, i: idler wave). In the stimulated scattering process, the momentum conservation law $k_{p}=k_{i}+k_{T}$ (noncollinear phase-matching condition, Figure 2) also holds. This leads to the angle-dispersive characteristics of the idler and terahertz waves. Thus, broadband terahertz waves are generated depending on the phase-matching angle. Generation of a coherent terahertz wave can be achieved by applying an optical resonator (in the case of the TPO) or injecting a "seed" for the idler wave (in the case of the is-TPG/TPO). Continuous and wide tunability is accomplished simply by changing the angle between the incident pump beam and the resonator axis or the seed beam.
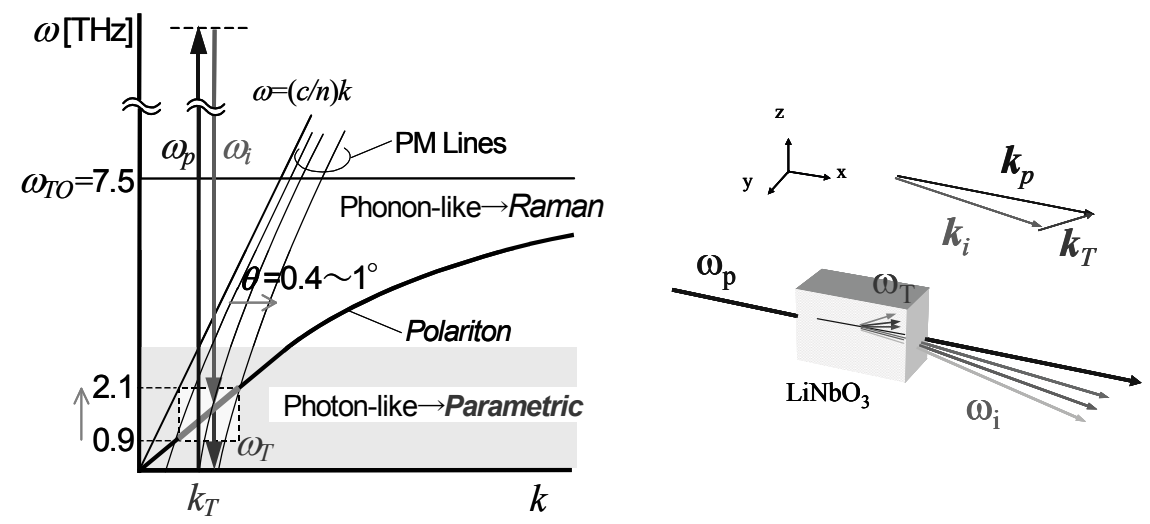

Fig. 2. Dispersion relation of the polariton. An elementary excitation is generated by the combination of a photon and a transverse optical phonon $\left(\omega_{\mathrm{TO}}\right)$. The polariton in the low energy region behaves like a photon at terahertz frequency. Due to the phase-matching condition as well as the energy conservation law which hold in the stimulated parametric process, tunable terahertz wave is obtained by the control of the wavevector $\boldsymbol{k}_{i}$. The right figure shows the noncolinear phase-matching condition.

The bandwidth of the TPG is decided by the parametric gain and absorption coefficients in the terahertz region. According to a plane-wave approach, analytical expressions of the exponential gain for the terahertz and idler wave are given by (Shikata et al, 2000; 2002)

$$
g_{T}=\frac{\alpha_{T}}{2}\left\{\sqrt{1+16 \cos \phi\left(\frac{g_{0}}{\alpha_{T}}\right)^{2}}-1\right\},
$$

where $a_{T}$ is the absorption coefficient of the terahertz wave in the nonlinear crystal. Parameter $\varphi$ is the phase-matching angle between the pump beam and the terahertz wave; $g_{0}$ is the parametric gain in the low-loss limit, and takes the form

$$
g_{0}=\sqrt{\frac{\pi \omega_{p} \omega_{i} I_{p}}{2 c^{3} n_{T} n_{i} n_{p}}} \chi_{p} \propto \sqrt{\omega_{i} \omega_{T} I_{p}},
$$




$$
\chi_{\rho}=d_{E}+\frac{S_{0} \omega_{0}^{2}}{\omega_{0}^{2}-\omega^{2}} d_{Q}
$$

where $I_{p}$ is the pump intensity, $n_{T}, n_{i}, n_{p}$ are the crystal refractive indices at the wavelengths of the terahertz wave, idler wave, and pump beam, respectively, $\omega_{0}$ is the resonance frequency of the lowest $\mathrm{A}_{1}$-mode, and $S_{0}$ is the oscillator strength. The nonlinear coefficients $d_{E}$ and $d_{Q}$ represent second- and third-order nonlinear processes, respectively. The absorption coefficient $a_{T}$ in the terahertz region is given by,

$$
\alpha_{T}=\frac{2 \omega}{c}\left|\operatorname{Im}\left(\sqrt{\varepsilon_{T}}\right)\right|
$$

where $\varepsilon_{T}$ is the dielectric constant of the nonlinear crystal.

Figure 3 shows the calculated gain and the absorption coefficient at several pump intensities. The gain curve has a broad bandwidth of around $3 \mathrm{THz}$, with a dip appearing at around 2.6 THz. This is because the low frequency modes of doped $\mathrm{MgO}$ in the $\mathrm{MgO}: \mathrm{LiNbO}_{3}$ work as a crystal lattice defects for $\mathrm{LiNbO}_{3}$.

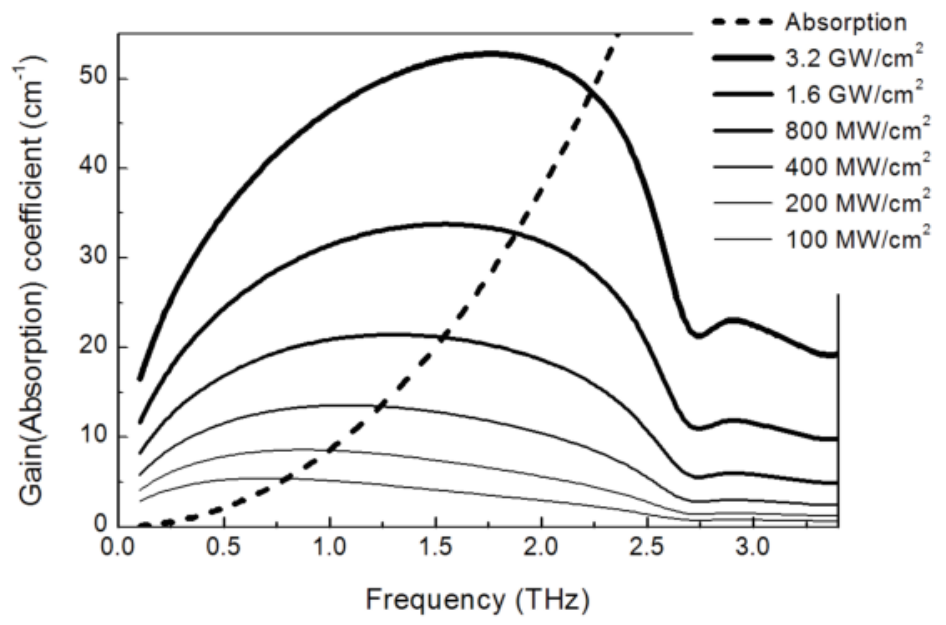

Fig. 3. Calculated gain and absorption coefficient.

\section{Terahertz-wave parametric generator (TPG)}

Broadband terahertz waves are generated by single-pass pumping, in a TPG. The linewidth of the terahertz wave emitted from the TPG is typically broad, about $1 \mathrm{THz}$. In addition, several applications are better suited to a broadband source (TPG) than to a nawwor linewidth source (TPO or is-TPG), such as tomographic imaging, interferometric spectroscopy, and diffuse reflection spectroscopy. Tomographic imaging and interferometric spectroscopy have to use a broadband source. The detection of scattered terahertz radiation strongly depends on the grain size of samples made of particles; using a broadband source reduces this effect. Also, the TPG is useful for many industrial applications such as transmission imaging for materials or food inspection. 
A TPG uses a quite simple configuration since it needs no resonator or seeder, as shown in Figure 4. The $\mathrm{MgO}: \mathrm{LiNbO}_{3}$ crystal used in the experiment was cut to the size $65(\mathrm{x}) \times 5(\mathrm{y}) \times$ $4(\mathrm{z}) \mathrm{mm}^{3}$. The $x$-surfaces at both ends were mirror polished and antireflection coated. The $y$-surface was also mirror polished, in order to minimize the coupling gap between the prism base and the crystal surface, and to prevent scattering of the pump beam. The pump beam passed through the crystal close to the $y$-surface, to minimize the travel distance of the terahertz wave inside the crystal.

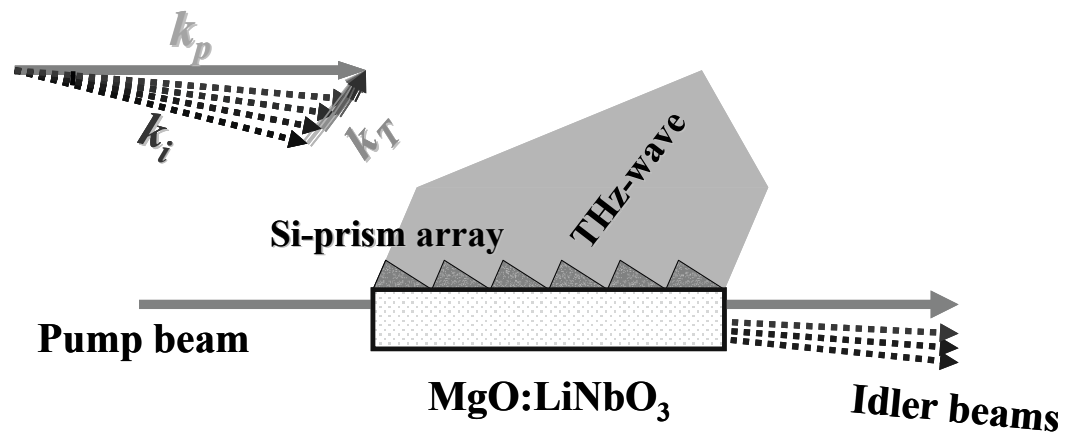

Fig. 4. A terahertz wave parametric generator with a Si-prism array. The Si-prism array was placed on the $y$-surface of the $\mathrm{MgO}: \mathrm{LiNbO}_{3}$ to increase the output and to reduce the diffraction angle of the terahertz wave by increasing the coupling area.

Most of the generated terahertz wave was absorbed or totally reflected inside the crystal due to the material's large absorption coefficient and large refractive index. Therefore, it was rather difficult to couple out the terahertz wave efficiently to the free space. We introduced a Si-prism coupler $(n \approx 3.4)$ to extract the terahertz wave generated inside a nonlinear crystal, thereby substantially improving the exit characteristics. The terahertz wave output energy, peak power and linewidth emitted from the TPG is typically $1 \mathrm{pJ} /$ pulse, $300 \mu \mathrm{W}$, and $1 \mathrm{THz}$ respectively.

\section{Terahertz-wave parametric oscillator (TPO)}

Coherent tunable terahertz waves can be generated by realizing a resonant cavity for the idler wave. This is the basic configuration of a TPO, and it consists of a Q-switched Nd:YAG laser, the nonlinear crystal placed inside the $15 \mathrm{~cm}$ long cavity, as shown in Figure 5. Both mirrors were half-area-coated, so that only the idler wave could resonate and the pump beam propagate through the uncoated area without scattering. The mirrors and a nonlinear crystal were mounted on a rotating stage, and tunability was obtained by rotating the stage slightly to vary the angle of the resonator with respect to the pump beam. The pump power and pulsewidth were $30 \mathrm{~mJ} /$ pulse and $25 \mathrm{~ns}$, respectively. The pump beam entered the $x$ surface of the crystal and passed through the $\mathrm{MgO}: \mathrm{LiNbO}_{3}$ crystal close to the surface of the Si-prism coupler to minimize the absorption loss of the terahertz wave. A near-infrared idler oscillation around $1.07 \mu \mathrm{m}$ was clearly recognized by its oscillating spot above a threshold pump power density of about $130 \mathrm{MW} / \mathrm{cm}^{2}$. The idler wave is amplified in the resonator consisting of flat mirrors with a half-area HR coating. The mirrors and crystal are installed on a precise, computer-controlled rotating stage for precise tuning. When the incident angle 
of the pump beam into the $\mathrm{MgO}: \mathrm{LiNbO}_{3}$ is varied between 3.13 and $0.84 \mathrm{deg}$, the angle between the pump wave and the idler wave in the crystal changes from 1.45 down to 0.39 $\mathrm{deg}$, whereas the angle between the terahertz wave and the idler wave changes from 67.3 down to $64.4 \mathrm{deg}$. With this slight variation in the phase-matching condition, the wavelength (frequency) of the terahertz wave could be tuned between 100 and $330 \mathrm{~mm} \mathrm{(3-}$ $0.9 \mathrm{THz}$ ); the corresponding idler wavelength changed from 1.075 down to $1.067 \mathrm{~mm}$. The terahertz wave radiation was monitored with a $4 \mathrm{~K}$ Si bolometer.

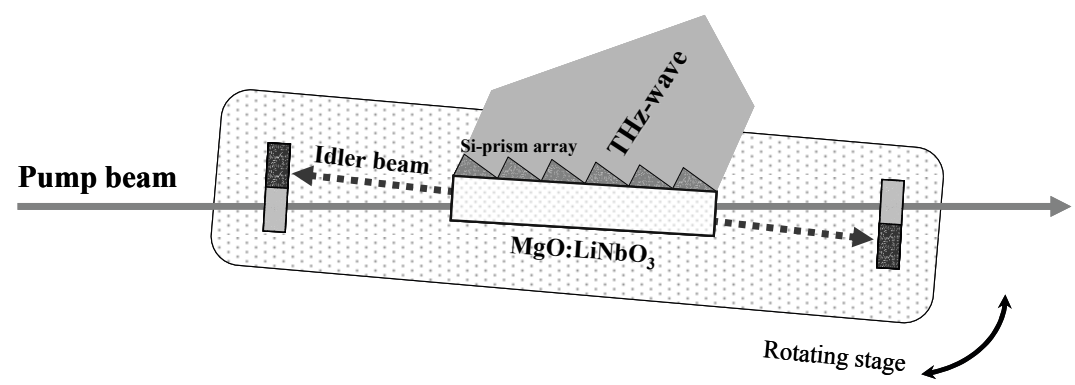

Fig. 5. TPO configuration. The TPO consists of a Q-switched Nd:YAG laser, a nonlinear crystal, and a parametric oscillator. The idler wave is amplified in the resonator consisting of flat mirrors with a half-area HR coating. The mirrors and crystal are installed on a precise, computer-controlled, rotating stage for fine tuning.

Typical input-output characteristics of a TPO are shown in Figure 6, in which the oscillation threshold was $18 \mathrm{~mJ} /$ pulse. With a pump power of $34 \mathrm{~mJ} /$ pulse, the output energy of terahertz wave from TPO was $192 \mathrm{pJ} /$ pulse ( $\cong 19 \mathrm{~mW}$ at the peak), calibrated using the sensitivity of the bolometer. Since the Si-bolometer output becomes saturated at approximately $5 \mathrm{pJ} /$ pulse, we used several sheets of thick paper as an attenuator after they were properly calibrated. The minimum sensitivity of the Si-bolometer is approximately 1 $\mathrm{fJ} /$ pulse, therefore, the dynamic range of measurement using the TPO as a source is $192 \mathrm{pJ} /$ $1 \mathrm{fJ}$, which exceeds $50 \mathrm{~dB}$.

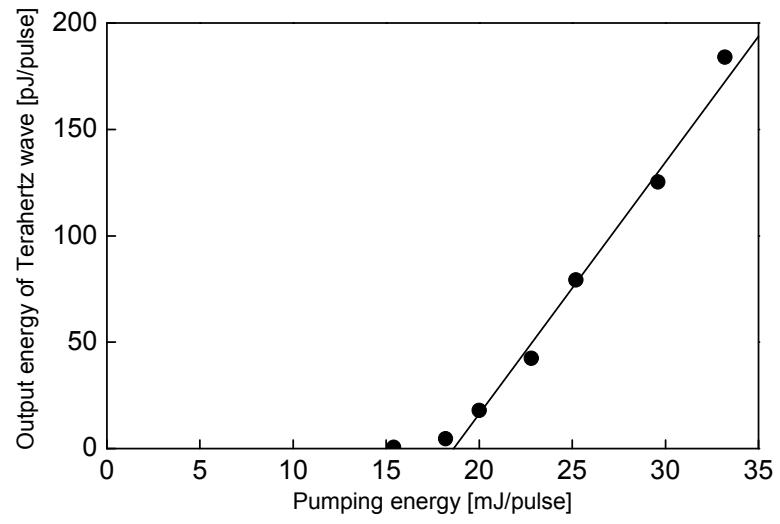

Fig. 6. Input-output characteristics of a terahertz wave parametric oscillator. 


\section{Injection-seeded Terahertz-wave parametric generator (is-TPG)}

The TPG spectrum was narrowed to the Fourier Transform limit of the pulse width by introducing an injection seeding for the idler wave. Figure 7 shows our experimental setup of the is-TPG. An array of seven Si-prism couplers was placed on the $y$-surface of the secondary $\mathrm{MgO}: \mathrm{LiNbO}_{3}$ crystal for efficient coupling of the terahertz wave. The pumping laser was a single longitudinal mode Q-switched Nd:YAG laser (wavelength: $1.064 \mu \mathrm{m}$; energy: < $50 \mathrm{~mJ} /$ pulse; pulsewidth: $15 \mathrm{~ns}$; beam profile: $\mathrm{TEM}_{00}$ ). The pump beam diameter was $0.8 \mathrm{~mm}$. The pump beam was almost normal to the crystal surfaces as it entered the crystals and passed through the crystal close to the $y$-surface. A continuous wave tunable diode laser (wavelength: 1.066-1.074 $\mu \mathrm{m}$; power: $50 \mathrm{~mW}$ ) was used as an injection seeder for the idler. Observation of the intense idler beam easily confirmed the injection-seeded terahertz wave generation. The polarizations of the pump, seed, idler, and terahertz waves were all parallel to the $z$-axis of the crystals. The terahertz wave output was measured with a $4 \mathrm{~K}$ Si bolometer.

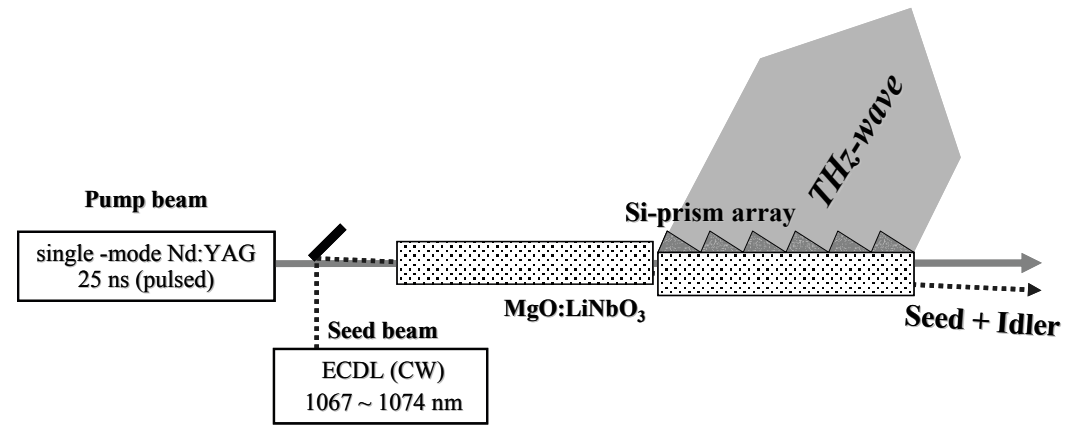

Fig. 7. Experimental setup of the is-TPG.

It was possible to tune the terahertz wavelength using an external cavity laser diode as a tunable seeder. A wide tunability, from 125 to $430 \mu \mathrm{m}$ (frequency: 0.7 to $2.4 \mathrm{THz}$ ), was achieved as shown in Figure 8 by changing simultaneously the seed wavelength and the seed incident angle. Open squares and closed circles indicate the tunability of the terahertz and idler waves, respectively. Both crystals were $\mathrm{MgO}_{\mathrm{LiNbO}}$ in this experiment. The wavelength of $430 \mu \mathrm{m}(0.7 \mathrm{THz})$ was the longest ever observed during our study of TPGs and TPOs. In the longer-wavelength region, the angle between the pump and idler becomes less than $1^{\circ}$; thus it is difficult for the TPO to oscillate only the idler inside the cavity without scattering the pump. In the shorter-wavelength region, the terahertz wave output is comparatively smaller than the idler wave output, due to the larger absorption loss inside the crystal.

The absorption spectrum of low-pressure ( $<1$ torr) water vapor was measured to demonstrate the continuous tunability and the high resolution of the is-TPG. The absorption gas cell used was an 87-cm-long stainless steel pipe with TPX windows at both ends. Figure 9 shows an example of measurements at around $1.92 \mathrm{THz}$, where two neighboring lines exist. Resolution of less than $100 \mathrm{MHz}\left(0.003 \mathrm{~cm}^{-1}\right)$ was clearly shown. In fact, it is not easy for FTIR spectrometers in the terahertz wave region to demonstrate a resolution better than $0.003 \mathrm{~cm}^{-1}$ because of the instability of the scanning mirror for more than a meter. The system is capable of continuous tuning at high spectral resolution in $4 \mathrm{GHz}$ segments 


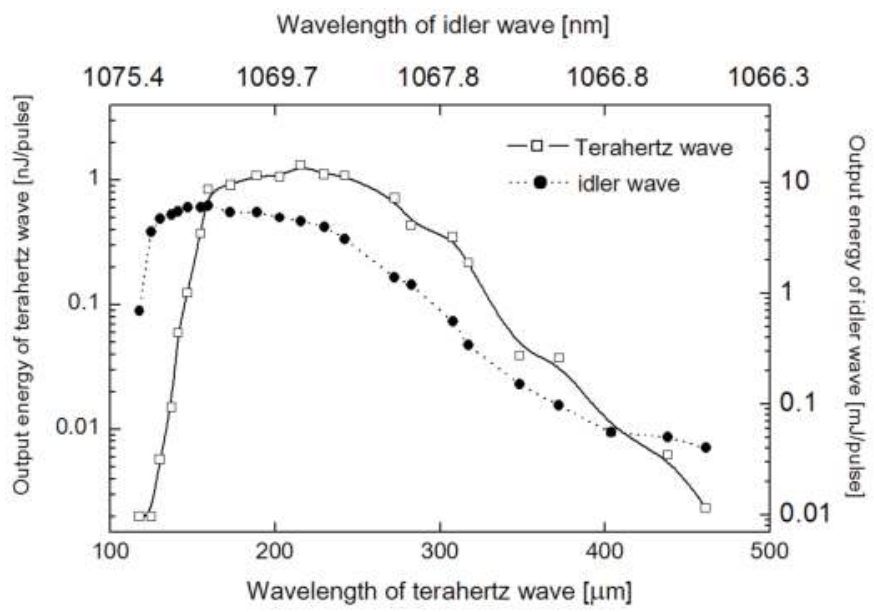

Fig. 8. Wide tunability of an is-TPG. Open squares and closed circles indicate the tunability of the $\mathrm{THz}$ and idler waves, respectively.

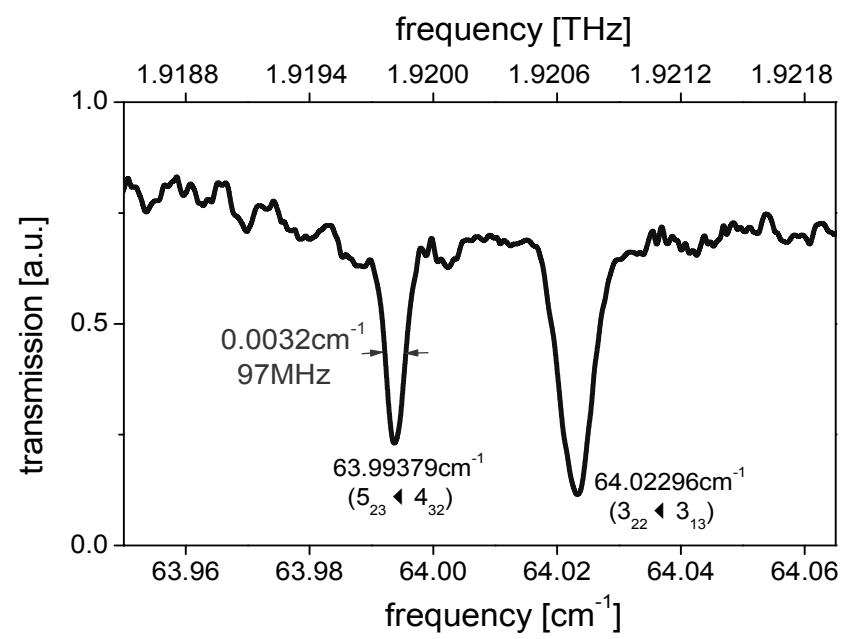

Fig. 9. An example of the absorption spectrum measurement of low-pressure ( $<1$ torr) water vapor at around $1.919 \mathrm{THz}$. Resolution of less than $100 \mathrm{MHz}(0.003 \mathrm{~cm}-1)$ was clearly demonstrated.

anywhere in the region from 0.7 to $2.4 \mathrm{THz}$. Since there is no cavity to be slaved, the continuous tuning is extendible, in principle, to the full tunability of the is-TPG by using a mode-hop-free seeder, such as a Littman-type external cavity diode laser.

The input-output characteristic of the terahertz wave from an is-TPG is shown in Figure10. The maximum conversion efficiency was achieved when the pump and seed beams almost fully overlapped at the incident surface of the first $\mathrm{MgO}: \mathrm{LiNbO}_{3}$ crystal. The maximum terahertz wave output of $1.3 \mathrm{~nJ} /$ pulse (peak power over $300 \mathrm{~mW}$ ) was obtained with a single-mode pump beam of $34 \mathrm{~mJ} /$ pulse and a seed beam of $50 \mathrm{~mW}$. To prevent saturating 
the Si bolometer, again we used several sheets of thick paper as an attenuator after calibrating them. In our previous studies, the maximum terahertz wave output from a conventional TPG and a TPO was 1 and $190 \mathrm{pJ} /$ pulse, respectively. The Si-bolometer became saturated at about $5 \mathrm{pJ} /$ pulse, so we used several thick calibrated sheets of paper as an attenuator. As the minimum sensitivity of the Si-bolometer is about $1 \mathrm{fJ} /$ pulse, the dynamic range of the is-TPG system was from $1.2 \mathrm{~nJ}$ down to $1 \mathrm{fJ}$, that is, $\sim 60 \mathrm{~dB}$, which is sufficient for most applications. The dynamic range can be significantly increased using a lock-in amplifier.

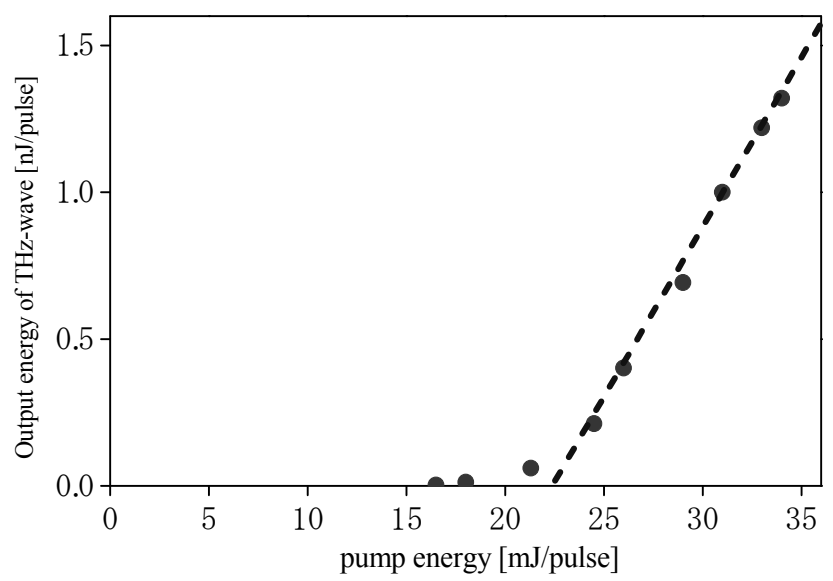

Fig. 10. Input-output characteristics of the is-TPG.

\section{Recent progress}

\subsection{Energy-enhanced TPG}

In this section, we report some recent developments of a TPG using a small pump source with a short pulse width and top-hat beam profile. In our experimental configuration, the output energy of the TPG is mainly limited by the damage threshold of the nonlinear crystal. We can generate high energy, broadband terahertz waves by using a short-pulsed pump beam with a top-hat beam profile which can provide high intensity pumping near the crystal surface without damaging the crystal.

The experimental apparatus, shown in figure 11, consists of a flash-lamp pumped Qswitched Nd:YAG laser, a lens, mirrors, and two nonlinear crystals. All components, except for the detector in figure 11, can be mounted on a $12 \times 22 \mathrm{~cm}$ breadboard. The small pump source has a short pulse width, of around $5 \mathrm{~ns}$. Its slight divergence is corrected by a lens placed at the output of the source. It has a top-hat profile with a beam diameter of $1.3 \mathrm{~mm}$ (full width at half maximum) on the first crystal. We used two 65-mm-long nonlinear $\mathrm{MgO}: \mathrm{LiNbO}_{3}$ crystals. Both crystal ends are antireflection coated for a wavelength of 1064 $\mathrm{nm}$. The gap between the two crystals is about $100 \mu \mathrm{m}$ in our experiment, which is short enough for the phase matching condition. A Si-prism array placed on the $y$-surface of the $\mathrm{MgO}: \mathrm{LiNbO}_{3}$ crystal acts as an efficient output coupler for the terahertz waves to avoid the total internal reflection of the terahertz waves on the output side crystal surface. 


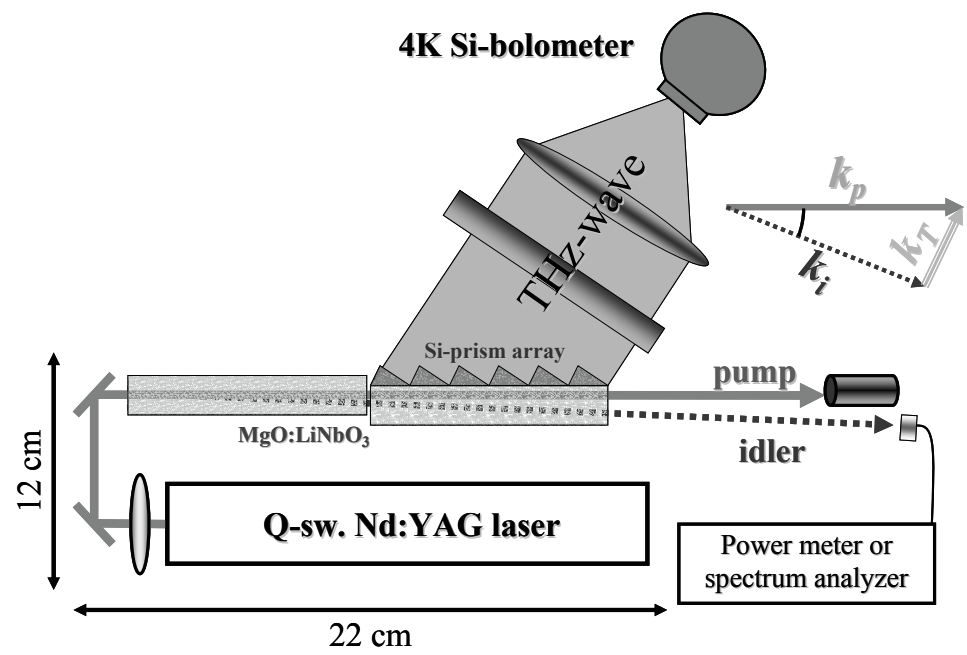

Fig. 11. Experimental setup of the energy-enhanced TPG.

For an efficient extraction of the terahertz waves, the pumped region inside the second crystal must be as close as possible to the Si-prism array, because of the large absorption coefficient of the $\mathrm{MgO}: \mathrm{LiNbO}_{3}$ crystal in the terahertz range. A top-hat beam profile is suitable for this purpose, since the high intensity region of the pump beam can be brought closer to the $y$-surface than in the case of a Gaussian beam. The distance between the $y$ surface and the beam center was precisely adjusted to obtain a maximum terahertz wave output, and it was approximately equal to the pump beam radius.

The terahertz wave output extracted through the Si-prism array was measured using a $4.2 \mathrm{~K}$ silicon bolometer, while the idler wave energy was measured using a pyroelectric detector. The minimum and maximum sensitivity levels of the bolometer are about $0.01 \mathrm{pJ}$ and $10 \mathrm{pJ}$ without any amplifier or attenuator. Attenuators were used when the detector was saturated; to cut diffused light from the pump and idler, a thick black polyethylene sheet was used.

Figure 12 shows the output energy/power (peak) of the terahertz wave as a function of the pump intensity. As the pump intensity is higher, the terahertz wave starts to be detected at a pump intensity of around $300 \mathrm{MW} / \mathrm{cm}^{2}(25 \mathrm{~mJ} /$ pulse $)$ then increase monotonically. The highest values obtained are $105 \mathrm{pJ} /$ pulse $(62 \mathrm{~mW}$ peak power) for the terahertz wave when the pump intensity is $830 \mathrm{MW} / \mathrm{cm}^{2}$ (66 $\mathrm{mJ} /$ pulse), which corresponds to a pump energy of $66 \mathrm{~mJ} /$ pulse. The output of terahertz wave appears to saturate when the pump intensity exceeds $750 \mathrm{MW} / \mathrm{cm}^{2}$ (60 mJ/pulse). Because higher intensity pumping leads to broader bandwidth as indicated by Eq. (1), however, the absorption coefficient for the terahertz wave rapidly increases in the high frequency range.

In previous TPG/TPO research, the crystal damage threshold was below the value of 200 $\mathrm{MW} / \mathrm{cm}^{2}$ for the pump beam intensity. With this report, by using a short-pulsewidth pump beam, the damage threshold is increased about 4 times. Moreover, the top-hat beam profile enables high intensity pumping especially close to the terahertz wave output surface, without any thermal damage to the crystal surface. These combined characteristics of pump beam yield 100 times more output energy of the terahertz wave. 


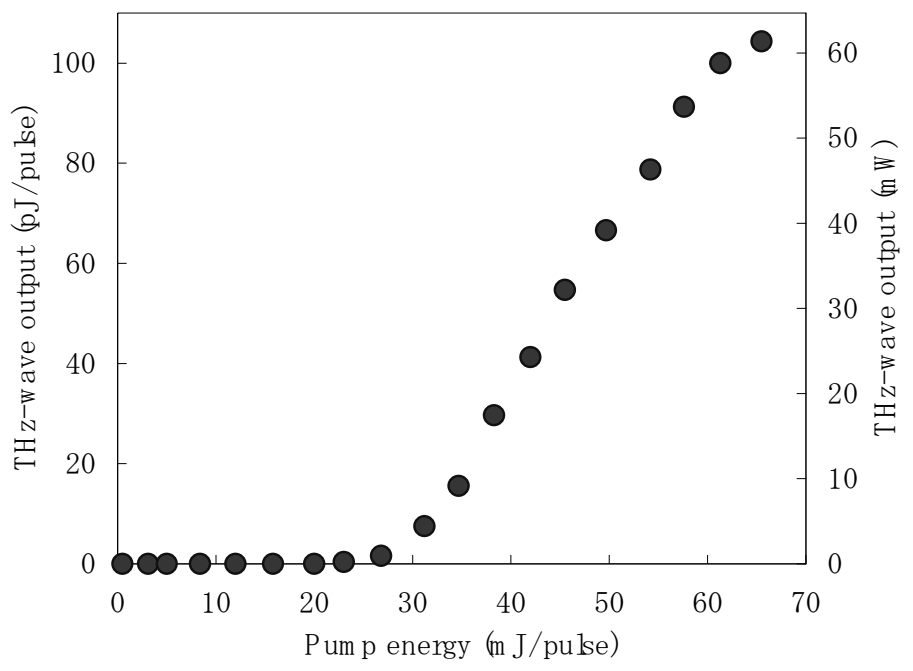

Fig. 12. The input-output characteristics of the energy-enhanced TPG.

Figure 13 shows the idler wave spectrum observed for varying pump energies. According to the noncollinear phase-matching condition, the propagating direction of the generated idler waves is slightly different from that of the pump beam, with an angle between them of around $1.5^{\circ}$ outside the crystal. As the pump energy increases, the idler wave spectrum covers a broader spectral region, especially towards longer wavelengths. At the maximum pump energy, the idler wave spectrum was found to cover the range $1067-1079 \mathrm{~nm}$. This spectrum corresponds to the terahertz wave frequency range $0.898-3.87 \mathrm{THz}(77.6-$ $334 \mu \mathrm{m})$. The measured spectrum is much broader than that observed in a previous report. The main reason for this broader spectrum might be the fact that the parametric gain could have broader bandwidth by higher pump energy as shown in Figure 3. The dip in the

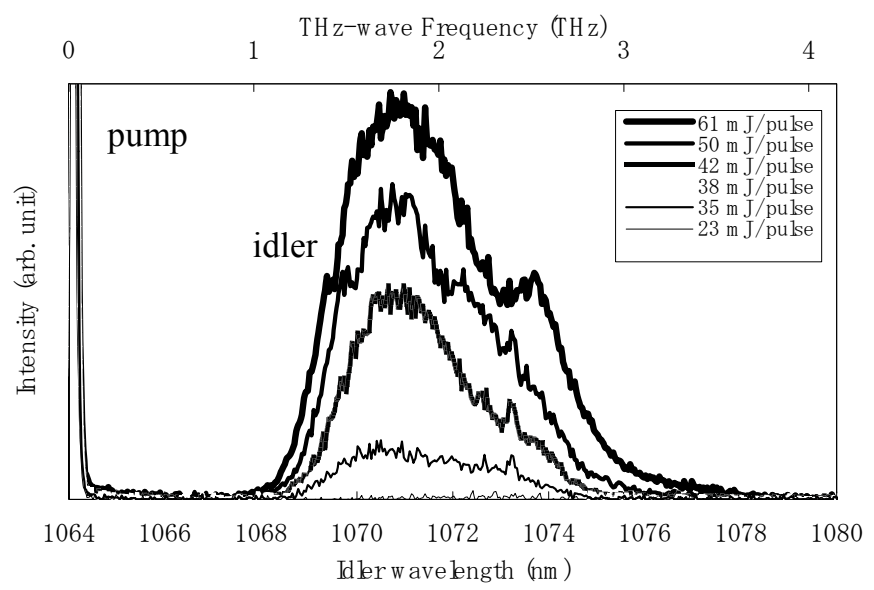

Fig. 13. Idler spectra at several pump energies. 
spectra around $1073 \mathrm{~nm}$ appears due to the $\mathrm{MgO}$ doping of the $\mathrm{LiNbO}_{3}$; the lattice defects produced by the $\mathrm{MgO}$ leads to additional peaks of the absorption coefficient $a_{T}$.

\subsection{Tunability-enhanced is-TPG}

We have enhanced the tunability of terahertz wave parametric generator using $\mathrm{MgO}: \mathrm{LiNbO}_{3}$ pumped by a sub-nanosecond, passively Q-switched, microchip Nd:YAG laser. This pump source allows high intensity pumping without damaging of the nonlinear crystal and generates a narrow linewidth and tunable terahertz wave with injection seeding by an external cavity diode laser for the idler wave. The high intensity pumping causes a gain curve broadening of the terahertz wave parametric generation, especially in the high frequency region.

The experimental setup, shown in Figure 14, consists of a pumping source (Microchip Nd:YAG laser), a seeding source (External Cavity Diode Laser) and the nonlinear crystal. The pump source is a diode end-pumped single-mode microchip $\mathrm{Nd}^{3+}$ :YAG laser, passively Q-switched by a $\mathrm{Cr}^{4+}$ :YAG saturable absorber. This microchip configuration enables the low order axial and transverse mode laser oscillation, whose linewidth is below $0.009 \mathrm{~nm}$. The laser delivers 1.1 MW peak power pulses $(530 \mu \mathrm{J} /$ pulse) with 430 ps pulse width at $100 \mathrm{~Hz}$ repetition rate with a $\mathrm{M}^{2}$ factor of 1.09 . This laser is free from the electric noise, unlike the active Q-switched lasers we used before. Additionally, this kind of fixed passively Qswitching allows us to obtain a stabilized peak power, with less than $\pm 2 \%$ power jitter (Pavel et al., 2001; Sakai et al., 2008 ). The pump beam diameter on the first crystal is $0.3 \mathrm{~mm}$ (full width at half maximum). We used two 65-mm-long nonlinear $\mathrm{MgO}: \mathrm{LiNbO}_{3}$ crystals. A silicon-prism array placed on the y surface of the second crystal acts as an efficient output coupler for the terahertz waves to avoid the total internal reflection of the terahertz waves on the crystal output side. For an efficient terahertz wave emission, the pumped region within the second crystal must be as close as possible to the silicon-prism array, because of the large absorption coefficient of the $\mathrm{MgO}: \mathrm{LiNbO}_{3}$ crystal in the terahertz range. The distance between the $y$-surface and the beam center was precisely adjusted to obtain a maximum terahertz wave output, and it was approximately equal to the pump beam radius. The terahertz wave output extracted through the silicon-prism array was measured using a $4.2 \mathrm{~K}$ silicon bolometer, while the idler-wave energy was measured using a pyroelectric detector.

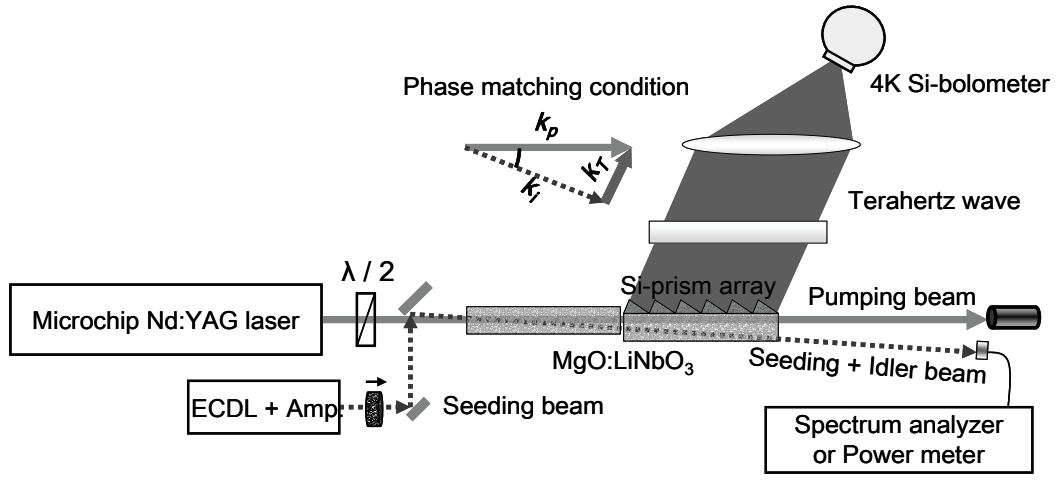

Fig. 14. Experimental setup of tenability-enhanced is-TPG. 
Figure 15 shows the idler wave spectrum when the pump intensity is $2.9 \mathrm{GW} / \mathrm{cm}^{2}$. According to the noncollinear phase matching condition, the propagating direction of the generated idler waves is slightly different from that of the pump beam, with an angle between them of around $1.5^{\circ}$ outside the crystal. The idler wave spectrum was found to cover the range 1069 - $1075 \mathrm{~nm}$, corresponding to the terahertz wave frequency range $1.4-2.9 \mathrm{THz}$ without seeding beam. Compared with a previous report, this is a shift to higher frequency. The dip in the spectra around $1073 \mathrm{~nm}$ appears due to the $\mathrm{MgO}$ doping of the $\mathrm{LiNbO}_{3}$; the lattice defects produced by the $\mathrm{MgO}$ leads to additional peaks of the absorption coefficient.

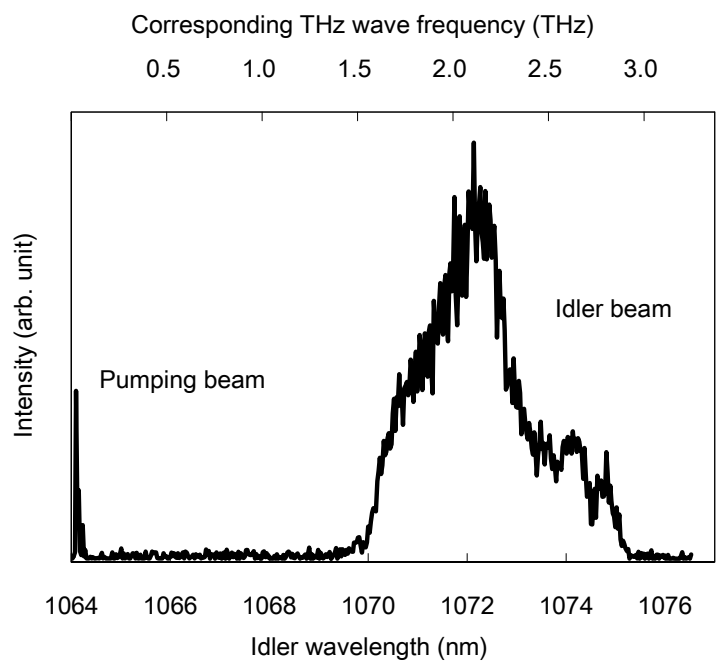

Fig. 15. Idler spectrum.

Figure 16 shows the time waveform of the terahertz wave output signals measured by the 4 $\mathrm{K}$ silicon bolometer. When we generate the terahertz wave without injection seeding to the idler wave, we observe a broadband terahertz wave with the peak power of about $1 \mathrm{~mW}$ (lower curve), however, after injection seeding, we observed a narrow linewidth terahertz wave with a peak power of about $20 \mathrm{~mW}$ (upper curve). This is about more than 100 times narrower and 20 times higher than when the seeding laser is cut off. In addition, the pulse width of this microchip laser is the shortest among our parametric sources.

It is possible to tune the terahertz frequency using an ECDL as a tunable seeder. When the pump intensity is $1.8 \mathrm{GW} / \mathrm{cm}^{2}$ (peak, energy of $650 \mu \mathrm{J} /$ pulse) and the seeding power is 80 $\mathrm{mW}(\mathrm{CW})$, a wide tunability from $0.9-3 \mathrm{THz}$ is observed, as shown in figure 6 by changing both the seed wavelength and the seed incident angle. The maximum output peak power of terahertz wave was about $100 \mathrm{~mW}$ at around $1.8 \mathrm{THz}$. The tuning curve has a broad bandwidth, with a dip appearing at around $2.7 \mathrm{THz}$. This is because the low frequency modes of doped $\mathrm{MgO}$ in the $\mathrm{MgO}: \mathrm{LiNbO}_{3}$ work as crystal lattice defects for $\mathrm{LiNbO}_{3} .{ }^{5}$

Figure 18 shows an example of wavelength and linewidth measurement by a scanning Fabry-Perot etalon consisting of two Ni metal-mesh plates with a $65 \mu \mathrm{m}$ grid. The displacement of one of the metal mesh plates corresponds directly to half of the wavelength. We observed a narrow linewidth terahertz wave with a wavelength of $140 \mu \mathrm{m}$ and peak power of about $60 \mathrm{~mW}$ by the $4 \mathrm{~K}$ silicon bolometer. The free spectral range (FSR) of the etalon was about $100 \mathrm{GHz}$, and the linewidth was measured to be less than $10 \mathrm{GHz}$. 


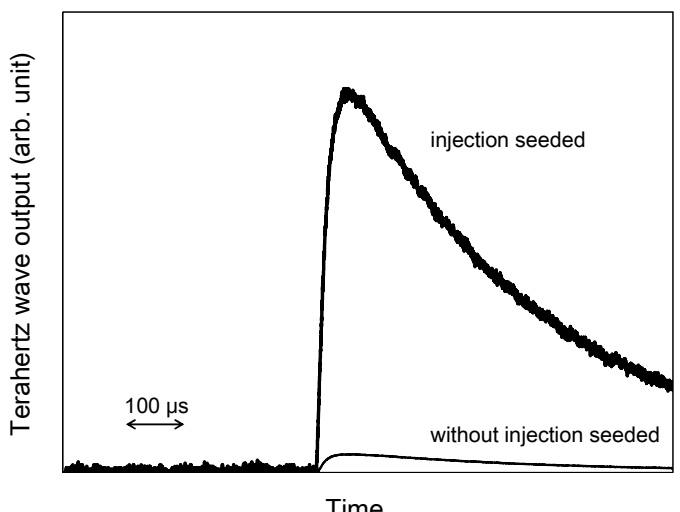

Time

Fig. 16. Time dependent terahertz wave output signals.

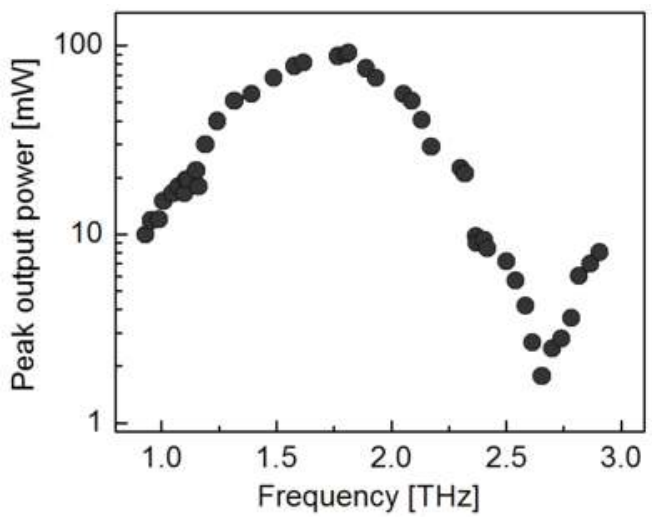

Fig. 17. Wide tunability of is-TPG with a dip at aroud $2.7 \mathrm{THz}$.

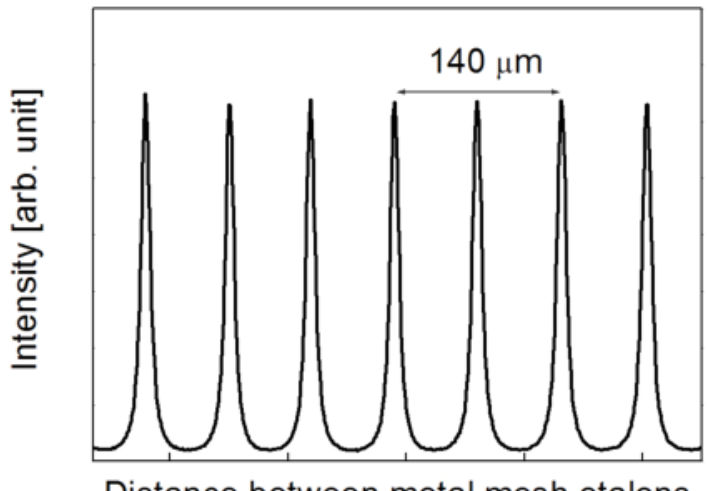

Distance between metal mesh etalons

Fig. 18. Example of the wavelength and linewidth measurement using the scanning FabryPerot etalon consisting of metal mesh plates. 


\section{Conclusion}

We reviewed terahertz wave parametric sources based on the optical parametric process. We have introduced several types of TPG, TPO, and is-TPG. Measurements on tunability, coherency, and power have been accomplished, proving this method to be suitable for many application fields. These include spectroscopy, communication, medical and biological applications, $\mathrm{THz}$ imaging, and so forth.

We also demonstrated output power enhancement of the TPG, while at the same time achieving a considerable downsizing of this terahertz source, all of which were realized by using a small pump source with a short pulse width and a top-hat beam profile. We measured a terahertz wave output energy of $105 \mathrm{pJ} /$ pulse, with a power peak at $62 \mathrm{~mW}$, and a broadband spectrum, extending from 0.9 to $3.8 \mathrm{THz}$. The new source is more than 100 times brighter and has a spectrum more than twice broader than previously reported.

In the next section, we demonstrated a compact and tunable terahertz wave source pumped by a microchip Nd:YAG laser. This source generates a narrow linewidth and high peak power terahertz wave by injection seeding for the idler wave. Using a microchip laser as the pumping source allowed high intensity pumping and the broadening of the tuning range towards the high frequency region. We could also observe a dip around $2.7 \mathrm{THz}$ in the tuning curve, as expected from the calculation.

Further improvement of our system is possible. As OPGs and OPOs have improved tremendously in the last decade, the use of TPGs and TPOs shows great potential to move towards a lower threshold, higher efficiency, and wider tunability. A lower threshold and a narrower linewidth can be expected using a nonlinear optical waveguide and a longer pump pulsewidth, respectively. Operation in other wavelength regions, through proper crystal selection, should also be possible. Success in this will prove the practicality of a new widely tunable THz-wave source, the IS-TPG, that will compete with free-electron lasers and p-Ge lasers. For tunable THz-wave applications, the simplicity of the wave source is an essential requirement since cumbersome systems do not encourage new experimental thoughts and ideas. Compared with the available sources, the present parametric method has significant advantages in compactness, tunability, and ease of handling.

\section{Acknowledgements}

The authors to thank Dr. Takayuki Shibuya, Dr. Hiroaki Minamide, Dr. Tomofumi Ikari, Prof. Takanari Yasui, Prof. Yuichi Ogawa, Prof. Jun-ichi Shikata, and Prof. Hiromasa Ito for useful discussions, and Prof. Takunori Taira, and Dr. Hiroshi Sakai for providing the microchip laser, Mr. Choichi Takyu for his excellent work coating the crystal surface, and Mr. Tetsuo Shoji for superbly polishing the crystals.

\section{References}

Hayashi, S.; Minamide, H.; Ikari, T.; Ogawa, Y.; Shikata, J.; Ito, H; Otani, C. \& Kawase, K. (2007). Output power enhancement of a palmtop terahertz-wave parametric generator. Appl. Opt., Vol. 46, 117 - 123, ISSN: 00036935.

Hayashi, S.; Shibuya, T.; Sakai, H.; Taira, T.; Otani, C.; Ogawa, Y.; \& Kawase, K. (2009). Tunability enhancement of a terahertz-wave parametric generator pumped by a microchip Nd:YAG laser. Appl. Opt., Vol. 48, No. 15, 2899-2902, ISSN: 00036935. 
Imai, K.; Kawase, K.; Shikata, J.; Minamide, H. \& Ito, H. (2001). Injection-seeded terahertzwave parametric oscillator. Appl. Phys. Lett., Vol. 78, 1026-1028, ISSN: 00036951.

Kawase, K.; Sato, M.; Taniuchi, T. \& Ito, H. (1996). Coherent tunable THz-wave generation from $\mathrm{LiNbO}_{3}$ with monolithic grating coupler. Appl. Phys. Lett., Vol. 68, 2483-2485, ISSN: 00036951.

Kawase, K.; Sato, M.; Nakamura, K.; Taniuchi, T. \& Ito, H. (1997). Unidirectional radiation of widely tunable $\mathrm{THz}$ wave using a prism coupler under noncollinear phase matching condition, Appl. Phys. Lett., Vol. 71, 753-755, ISSN: 00036951.

Kawase, K.; Shikata, J; Minamide, H.; Imai, K. \& Ito, H. (2001). Arrayed silicon prism coupler for a terahertz-wave parametric oscillator. Appl. Opt., Vol. 40, 1423-1426, ISSN: 00036935.

Kawase, K.; Shikata, J.; Imai, K. \& Ito, H. (2001). Transformlimited, narrow-linewidth, terahertz-wave parametric generator. Appl. Phys. Lett., Vol. 78, 2819-2821, ISSN: 00036951.

Kawase, K.; Minamide, H.; Imai, K.; Shikata, J. \& Ito, H. (2002). Injection-seeded terahertzwave parametric generator with wide tenability. Appl. Phys. Lett., Vol. 80, 195-197, ISSN: 00036951.

Kawase, K.; Shikata, J. \& Ito, H. (2002). Terahertz-wave parametric source. J. Phys. D, Vol. 35, R1-R14, ISSN 0022-3727.

Pavel, N.; Saikawa, J.; Kurimura, S. \& Taira, T. (2001). High Average Power Diode EndPumped Composite Nd:YAG Laser Passively Q-switched by $\mathrm{Cr}^{4+}$ :YAG Saturable Absorber. Jpn. J. Appl. Phys., Vol. 40, pt. 1, no. 3A, 1253-1259, ISSN:0021-4922.

Piestrup, M. A.; Fleming, R. N. \& Pantell, R. H. (1975). Continuously tunable submillimeter wave source. Appl. Phys. Lett., Vol. 26, 418-421, ISSN: 00036951.

Sakai, H.; Kan, H. \& Taira, T. (2008). > 1 MW peak power single-mode high-brightness passively Q-switched $\mathrm{Nd}^{3+}$ :YAG microchip laser. Opt. Exp., Vol. 16, 19891-19899, ISSN: 1094-4087.

Sato, A.; Kawase, K.; Minamide, H.; Wada, S. \& Ito H. (2001). Tabletop terahertz-wave parametric generator using a compact, diode-pumped Nd:YAG laser. Rev. Sci. Instrum., Vol. 72, 3501-3504, ISSN: 0034-6748.

Shikata, J.; Kawase, K.; Karino, K.; Taniuchi, T. \& Ito H. (2000). Tunable terahertz-wave parametric oscillators using $\mathrm{LiNbO}_{3}$ and $\mathrm{MgO}: \mathrm{LiNbO}_{3}$ crystals. IEEE Trans. Microwave Theory Tech., Vol. 48, 653-661, ISSN: 0018-9480.

Shikata, J.; Kawase, K.; Taniuchi, T. \& Ito, H. (2002). Fouriertransform spectrometer with a terahertz-wave parametric generator. Jpn. J. Appl. Phys., Vol. 41, 134-138, ISSN:0021-4922.

Shoji, I.; Kondo, T.; Kitamoto, A.; Shirane, M.\& Ito,R. (1997). Absolute scale of second-order nonlinear-optical coefficients. J. Opt. Soc. Am. B, Vol. 14, 2268-2294, ISSN: 0740-3224.

Smith, P. R.; Auston, D. H. \& Nuss, M. C. (1988). Subpicosecond photoconducting dipole antennas. IEEE J. Quantum Electron., Vol. 24, 255-260, ISSN: 0018-9197.

Sussman, S. S. (1970). Tunable Scattering from Transverse Optical Modes in Lithium Niobate. Microwave Laboratory Report, No. 1851 (Stanford University).

Tonouchi, M. (2007), Cutting-edge terahertz technology, Nature Photonics, Vol. 1, 97-105, ISSN: $1749-4885$.

Zhang, X. C.; Hu, B. B.; Darrow J. T. \& Auston D. H. (1990). Generation of femtosecond electromagnetic pulses from semiconductor surfaces. Appl. Phys. Lett., Vol 56, 1011-1013, ISSN: 00036951. 


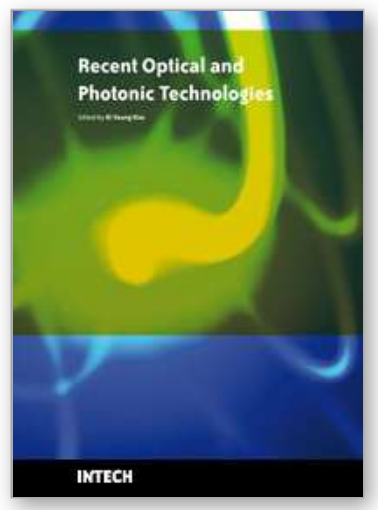

\author{
Recent Optical and Photonic Technologies \\ Edited by Ki Young Kim
}

ISBN 978-953-7619-71-8

Hard cover, 450 pages

Publisher InTech

Published online 01, January, 2010

Published in print edition January, 2010

Research and development in modern optical and photonic technologies have witnessed quite fast growing advancements in various fundamental and application areas due to availability of novel fabrication and measurement techniques, advanced numerical simulation tools and methods, as well as due to the increasing practical demands. The recent advancements have also been accompanied by the appearance of various interdisciplinary topics. The book attempts to put together state-of-the-art research and development in optical and photonic technologies. It consists of 21 chapters that focus on interesting four topics of photonic crystals (first 5 chapters), THz techniques and applications (next 7 chapters), nanoscale optical techniques and applications (next 5 chapters), and optical trapping and manipulation (last 4 chapters), in which a fundamental theory, numerical simulation techniques, measurement techniques and methods, and various application examples are considered. This book deals with recent and advanced research results and comprehensive reviews on optical and photonic technologies covering the aforementioned topics. I believe that the advanced techniques and research described here may also be applicable to other contemporary research areas in optical and photonic technologies. Thus, I hope the readers will be inspired to start or to improve further their own research and technologies and to expand potential applications. I would like to express my sincere gratitude to all the authors for their outstanding contributions to this book.

\title{
How to reference
}

In order to correctly reference this scholarly work, feel free to copy and paste the following:

Shin'ichiro Hayashi and Kodo Kawase (2010). Terahertz-Wave Parametric Sources, Recent Optical and Photonic Technologies, Ki Young Kim (Ed.), ISBN: 978-953-7619-71-8, InTech, Available from: http://www.intechopen.com/books/recent-optical-and-photonic-technologies/terahertz-wave-parametricsources

\section{INTECH}

open science | open minds

\section{InTech Europe}

University Campus STeP Ri

Slavka Krautzeka 83/A

51000 Rijeka, Croatia

Phone: +385 (51) 770447

Fax: +385 (51) 686166

\section{InTech China}

Unit 405, Office Block, Hotel Equatorial Shanghai

No.65, Yan An Road (West), Shanghai, 200040, China 中国上海市延安西路65号上海国际贵都大饭店办公楼 405 单元

Phone: +86-21-62489820

Fax: +86-21-62489821 
www.intechopen.com 
(C) 2010 The Author(s). Licensee IntechOpen. This chapter is distributed under the terms of the Creative Commons Attribution-NonCommercialShareAlike-3.0 License, which permits use, distribution and reproduction for non-commercial purposes, provided the original is properly cited and derivative works building on this content are distributed under the same license. 